\title{
Comportamiento termomecánico y termoquímico de ladrillos refractarios $\mathrm{MgO}-\mathrm{C}$
}

\section{Thermomechanical and thermochemical behavior of MgO-C refractory bricks}

Presentación: 6 y 7 de octubre del 2020

\section{Doctorando:}

\section{Yamila Soledad Lagorio}

Centro de Desarrollo y Tecnología de Materiales (DEYTEMA), Departamento Metalurgia, Facultad Regional San Nicolás, Universidad Tecnológica Nacional, Argentina

ylagorio@frsn.utn.edu.ar

\section{Director:}

\section{Edgardo Roque Benavidez}

\section{Resumen}

El desarrollo tecnológico de los ladrillos de MgO-C y su posición en el mercado, genera un gran interés en continuar estudiando ciertos temas que aún son de discusión. El desarrollo del tema de esta tesis es de utilidad, tanto para el sector académico como el industrial, en la generación de conocimiento integral de los refractarios $\mathrm{MgO}-\mathrm{C}$. El objetivo es estudiar el efecto que tiene la calidad de las materias primas en el comportamiento químico, mecánico y térmico de estos materiales. Para esto se formularon ladrillos con dos calidades de agregados de magnesia (electrofundidos y sinterizados) y con diferentes grados de pureza (entre 97,6 - 99,1\% Mg0). Los materiales para este estudio se prepararon a partir de $90 \%$ en peso de magnesia (Mg0) y 8,2\% en peso de grafito (C), que se completó con la adición de antioxidantes y resina ligante. Se determinaron: (i) composición química y mineralógica, (ii) análisis microestructural, (iii) densidad y porosidad (aparente y total), (iv) resistencia al ataque químico por escorias a 1600 ${ }^{\circ} \mathrm{C}$, (v) comportamiento mecánico en compresión a temperatura ambiente y a $1400{ }^{\circ} \mathrm{C}$, y (vi) evolución térmica hasta $1400^{\circ} \mathrm{C}$.

Palabras clave: Refractarios, MgO-C, Microestructura, Propiedades mecánicas, Propiedades térmicas, Corrosión

\begin{abstract}
The technological development of $\mathrm{MgO}-\mathrm{C}$ bricks and their position in the market generate great interest in continuing to study certain topics that are still under discussion, despite the fact that these materials have been extensively investigated. The development of this thesis is useful, to the academic than industrial sectors, in the generation of comprehensive knowledge of $\mathrm{MgO}-\mathrm{C}$ refractories. The objective of the thesis is to study the raw materials quality effect has on the chemical, mechanical and thermal behavior of these materials. For this, bricks were formulated with two qualities of magnesia aggregates (electrofused and sintered) and with different degrees of purity (between 97.6 - 99.1\% Mg0). The materials for this study were prepared from $90 \mathrm{wt} \%$ of magnesia (MgO) and 8.2 $\mathrm{wt} \%$ of graphite (C), completed with the addition of antioxidants and binder resin. Chemical and mineralogical composition, microstructural analysis, density and porosity (apparent and total), resistance to chemical attack by slags at $1600{ }^{\circ} \mathrm{C}$, mechanical behavior in compression at room temperature and $1400^{\circ} \mathrm{C}$, and thermal evolution up to 1400 ${ }^{\circ} \mathrm{C}$ were determined.
\end{abstract}

Keywords: Refractories, MgO-C, Microstructure, Mechanical Properties, Thermal Properties, Corrosion 


\section{Introducción}

Los refractarios, considerados facilitadores de otros materiales, son usados en procesos industriales, en donde prevalecen condiciones de elevadas temperaturas y medios corrosivos tales como metales y vidrios fundidos, escorias líquidas y gases calientes. Los refractarios son fundamentales en las industrias, tales como la siderurgia, la petroquímica, la producción de metales no ferrosos, de vidrio, de cemento y de cerámica, en las plantas de generación de energía e incineradoras de residuos, entre otras.

En la actualidad la industria siderúrgica es la mayor consumidora de materiales refractarios, empleándolos en la construcción y recubrimiento de hornos, cucharas y vagones termos, entre otras instalaciones. Esto impacta en los costos de las plantas y es allí donde nace la necesidad de llevar a cabo una selección adecuada de los refractarios. El uso de ladrillos refractarios de $\mathrm{MgO}-\mathrm{C}$ en la industria del acero se generalizó a principios de los años ochenta, dando lugar a la mejora del proceso metalúrgico, reduciendo drásticamente del consumo de refractario por tonelada de acero, al mismo tiempo, se garantizó la continuidad y estabilidad de los procesos de fusión (Xiao J. et al, 2019). La fabricación de acero se caracteriza por la continua demanda de aumentar la pureza y tener especificaciones de composición cada vez más estrictas, y que por esta razón requiere condiciones químicas, térmicas y mecánicas extremas, que exigen contar con materiales de alto desempeño.

El contacto con líquidos corrosivos, elevadas temperaturas, partículas en movimiento y gases calientes, son algunas de las condiciones a las que son sometidos los materiales refractarios en servicio. En muchos casos, la combinación de la corrosión junto con solicitaciones mecánicas, choque térmico y el desgaste por erosión y abrasión, provocan el fallo y disminución de la vida útil del refractario, lo cual lleva a su deterioro y reemplazo. Por este motivo, con el objetivo de evaluar el desempeño en servicio y establecer criterios de diseño del material, el conocimiento de las propiedades (térmicas, químicas y mecánicas) de los refractarios de $\mathrm{MgO}-\mathrm{C}$, a alta temperatura es indispensable.

El presente trabajo tiene como objetivo estudiar el comportamiento integral (térmico-mecánico-químico) de ladrillos refractarios de $\mathrm{MgO}-\mathrm{C}$ conformados con distintas calidades de magnesia, bajo condiciones similares a las de proceso. Los objetivos específicos son: (i) identificar los diferentes mecanismos de corrosión que actúan sobre los distintos ladrillos de MgO-C ante la presencia de escoria, (ii) evaluar el comportamiento mecánico, específicamente la resistencia bajo cargas de compresión, de los ladrillos refractarios, en diferentes atmósferas y temperaturas, (iii) determinar su evolución estructural con la temperatura y (iv) comparar los resultados obtenidos en los ladrillos de $\mathrm{MgO}-\mathrm{C}$ estudiados en la presente tesis con ladrillos comerciales.

\section{Desarrollo}

Para esta tesis se conformaron siete ladrillos, identificados como EFSZ, SZ1, SZ2, SZ3, EF1, EF2 y EF3, los cuales fueron formulados con un contenido de $\mathrm{MgO}$ del 90\% y 8,2\% de material carbonoso, difiriendo en la calidad (MgO electrofundida o sinterizada) y pureza de las magnesias. Las muestras EF contienen 100\% MgO electrofundida, las SZ se componen de $100 \% \mathrm{MgO}$ sinterizada y la muestra EFSZ de $40 \% \mathrm{MgO}$ sinterizada y $60 \% \mathrm{MgO}$ electrofundida. Es importante destacar que los materiales que se estudiarán en esta tesis no son materiales comerciales.

Las propiedades físicas (porosidad y densidad aparente) de los materiales evaluados en estado de recepción, se determinaron aplicando el método de Arquímedes, usando querosene como líquido de inmersión. Además, se evaluó la porosidad real o total de los ladrillos a partir de medidas de masa, utilizando querosene como fluido y empleando un recipiente de volumen calibrado (picnómetro). El ensayo se realizó por duplicado sobre $\approx 20 \mathrm{~g}$ de muestra en polvo, obtenido a partir de la trituración, molienda, y posterior tamizado a través de malla ASTM $\mathrm{N}^{\circ} 70(<0,212 \mathrm{~mm})$.

La resistencia al ataque químico se estudió mediante un ensayo de corrosión estática (denominado "cup test"), se confeccionaron crisoles de las siguientes dimensiones: base $=70 \times 70 \mathrm{~mm}^{2}$ y altura $=70 \mathrm{~mm}$, con un orificio central de $30 \mathrm{~mm}$ de diámetro y $40 \mathrm{~mm}$ de profundidad. En cada orificio se colocó $35 \mathrm{~g}$ de escoria de convertidor LD. El ensayo de "cup test" se realizó a $1600^{\circ} \mathrm{C}$ ( 2 horas) en atmósfera de aire.

Mediante fluorescencia de rayos X (FRX) se determinaron los contenidos de los principales óxidos presentes en las magnesias y la composición química de la escoria.

Para la evaluación mecánica se determinaron curvas tensión-deformación en compresión (CSS) sobre probetas cilíndricas (30 mm de diámetro y $50 \mathrm{~mm}$ de altura). Se utilizó una máquina universal de ensayos mecánicos servohidráulica (Instron, modelo 8501) la deformación de la probeta se determinó mediante un extensómetro axial

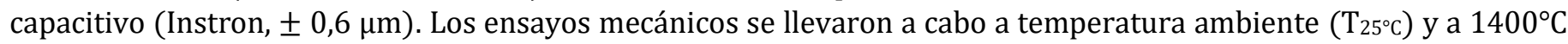
(en argón), con una velocidad de desplazamiento del actuador de $0,1 \mathrm{~mm} / \mathrm{min}$ hasta la rotura de la probeta. 
Para el examen microestructural, se cortaron, embutieron y pulieron muestras de los tres ladrillos en estado de recepción y post-ensayo de corrosión. Para el análisis por microscopía óptica (OM) se utilizó una lupa estereoscópica Olympus SZ61, un microscopio Carl Zeiss Axiotech mientras que para el estudio microestructural electrónico se empleó un microscopio electrónico de barrido (SEM) FEI Quanta 200 con posibilidad de análisis dispersivo de energía (EDS). Al análisis estructural de las muestras se añade la identificación de las fases cristalinas, de las muestras en estado de recepción y post-ensayo de compresión a $1400^{\circ} \mathrm{C}$, aplicando la técnica de Difracción de Rayos X (DRX), mediante el empleo de un difractómetro marca Philips X'Pert. Por otro lado, para completar el análisis estructural se determinó el tamaño de grano $\left(\mathrm{S}_{\mathrm{sG}}\right)$ de las partículas de magnesia electrofundida, aplicando un método lineal de medición utilizando las imágenes obtenidas de la microscopia óptica de las muestras vírgenes y un software de tratamiento de imágenes.

Para evaluar la expansión térmica se utilizó un dilatómetro horizontal (Theta, Dilatronic II), con el cual se ensayaron muestras entre $15-20 \mathrm{~mm}$ de longitud inicial. La velocidad de calentamiento y enfriamiento fue de $5^{\circ} \mathrm{C} / \mathrm{min}$, en atmósfera de argón, hasta una temperatura de $1400^{\circ} \mathrm{C}$.

\section{Resultados}

De acuerdo a la composición química (expresada en los contenidos principales de óxidos presentes) de la magnesia utilizada en su conformación, tabla 1, el ladrillo SZ1 presenta un mayor grado de pureza $(99,1 \%)$ de los granos de MgO, seguido por SZ2 y EF3 (98,99\%).

\begin{tabular}{|c|c|c|c|c|c|c|c|c|}
\hline Óxido & $\mathbf{M g O}$ & $\mathbf{A l}_{\mathbf{2}} \mathbf{O}_{\mathbf{3}}$ & $\mathbf{C a O}$ & $\mathbf{F e}_{\mathbf{2}} \mathbf{O}_{\mathbf{3}}$ & $\mathbf{S i O}_{\mathbf{2}}$ & $\mathbf{N a}_{\mathbf{2}} \mathbf{O}$ & $\mathbf{K}_{\mathbf{2}} \mathbf{O}$ & $\mathbf{P}_{\mathbf{2}} \mathbf{O}_{\mathbf{5}}$ \\
\hline EFSZ & 98,56 & 0,87 & 0,13 & 0,53 & 0,08 & 0,02 & 0,02 & 0,08 \\
\hline $\mathbf{S Z 1}$ & 99,1 & 0,11 & 0,58 & 0,45 & 0,02 & 0,02 & 0,02 & 0,05 \\
\hline $\mathbf{S Z 2}$ & 98,99 & 0,16 & 0,47 & 0,48 & 0,02 & 0,02 & 0,02 & 0,04 \\
\hline $\mathbf{S Z 3}$ & 98,42 & 0,06 & 1,21 & 0,22 & 0,12 & 0,02 & 0,02 & 0,03 \\
\hline EF1 & 97,61 & 1,09 & 0,73 & 0,51 & 0,08 & 0,02 & 0,02 & 0,11 \\
\hline EF2 & 98,2 & 1,06 & 0,2 & 0,58 & 0,06 & 0,02 & 0,02 & 0,1 \\
\hline EF3 & 98,99 & 0,78 & 0,11 & 0,48 & 0,04 & 0,02 & 0,02 & 0,08 \\
\hline
\end{tabular}

Tabla 1: composición química de las magnesias que conforman los ladrillos refractarios.

En la tabla 2 se presentan los valores de densidad aparente $\left(\rho_{A}\right)$, porosidad aparente $\left(\mathrm{P}_{\mathrm{A}}\right)$, porosidad total $\left(\mathrm{P}_{\mathrm{T}}\right)$ y porosidad cerrada $\left(\mathrm{P}_{\mathrm{C}}\right)$ de los materiales estudiados. La porosidad cerrada $\left(\mathrm{P}_{\mathrm{C}}\right)$ se calcula como: $P_{C}=P_{T}-P_{A}$.

\begin{tabular}{|c|c|c|c|c|c|c|c|}
\hline Muestra & EFSZ & SZ1 & SZ2 & SZ3 & EF1 & EF2 & EF3 \\
\hline $\mathbf{P A}_{\mathbf{A}}\left(\mathbf{g} / \mathbf{c m}^{\mathbf{3}}\right)$ & 2,86 & 2,84 & 2,88 & 2,91 & 2,91 & 2,95 & 2,95 \\
\hline $\mathbf{P A}_{\mathbf{A}}(\mathbf{\%})$ & 6,07 & 5,39 & 3,97 & 4,76 & 5,2 & 3,2 & 3,62 \\
\hline $\mathbf{P T}(\%)$ & 9,21 & 13,38 & 13,06 & 9,02 & 7,54 & 7,06 & 6,18 \\
\hline $\mathbf{P c}(\%)$ & 3,14 & 7,99 & 9,09 & 4,26 & 2,34 & 3,86 & 2,56 \\
\hline
\end{tabular}

Tabla 2: Valores de densidad aparente y de porosidad los ladrillos refractarios.

La corrosión debido a las escorias se debe en gran parte a la porosidad del material, de tal modo el grado de corrosión crece con el aumento de la porosidad. De la misma manera, el comportamiento mecánico (elástico) de los ladrillos de $\mathrm{MgO}-\mathrm{C}$, se ven afectados por el nivel de porosidad de los materiales.

En la figura 1 (a, b y c) se presentan imágenes de microscopia óptica (aumento 50x) de tres ladrillos estudiados (EF2, EFSZ y SZ1), donde podremos observar las diferencias estructurales de los ladrillos. En las mismas se destacan los granos de magnesia sinterizada (MSZ) y electrofundida (MEF), tanto en la fase de agregados (partículas $>500 \mu \mathrm{m}$ ) como en la matriz. En la matriz además se pueden observar partículas blancas brillantes que, de acuerdo con el análisis por EDS realizado, correspondería a la presencia de aluminio (Al) utilizado como antioxidante (marcadas como A). Por otro lado, con el análisis por SEM/EDS se confirma la presencia de Si como otro tipo de antioxidante adicionado a los ladrillos. También, en la matriz, es posible visualizar la presencia de grafito, en forma de láminas/hojuelas (flakes) de tonalidad gris (G). 


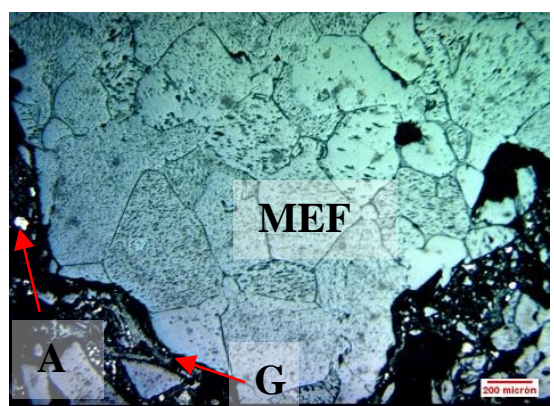

(a)

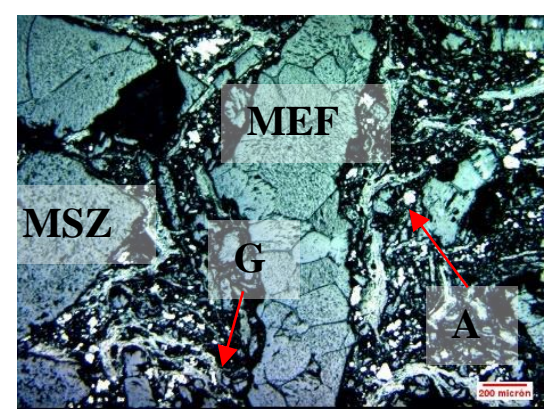

(b)

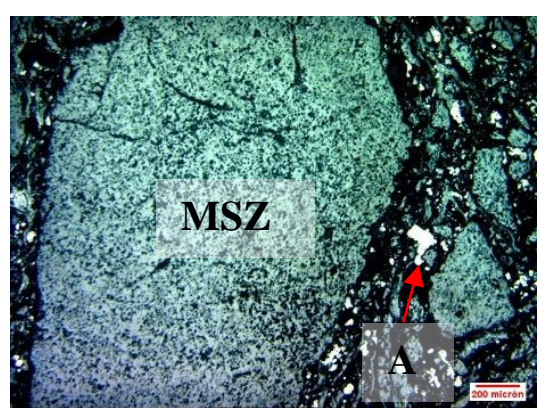

(c)

Figura 1: Microestructura de los ladrillos en estado de recepción: (a) ladrillo EF2, (b) ladrillo EFSZ y (c) ladrillo SZ1.

En la tabla 3 se presentan los valores de las mediciones del tamaño de grano $\left(\mathrm{S}_{\mathrm{SG}}\right)$ de los agregados de magnesia electrofundida, utilizando el método lineal de medición. Además, mediante un análisis puntual de EDS, fue posible estimar la composición de las segundas fases y la relación $\mathrm{CaO} / \mathrm{SiO}_{2}(\mathrm{C} / \mathrm{S})$ presentes en los bordes de grano y puntos triples de las $\mathrm{MgO}$ electrofundidas.

\begin{tabular}{|c|c|c|c|}
\hline Muestra & EF1 & EF2 & EF3 \\
\hline $\mathrm{S}_{\mathrm{SG}}(\mu \mathrm{m})$ & 112,5 & 176,6 & 245,8 \\
\hline $\mathrm{CaO} / \mathrm{SiO}_{2}$ & 0,67 & 0,91 & 1,50 \\
\hline
\end{tabular}

Tabla 3: Valores de tamaño de grano de partículas y relación $\mathrm{C} / \mathrm{S}$

Una menor relación $\mathrm{CaO} / \mathrm{SiO}_{2}$ entre los granos de $\mathrm{MgO}$ genera presencia de fases liquidas a menor temperatura. $\mathrm{Si}$ se tiene un menor tamaño de granos en las partículas de MgO, se tiene una mayor cantidad de bordes de grano, por consiguiente, una mayor cantidad de segundas fases (de bajas temperaturas de fusión) lo que provocaría una disminución en la resistencia a la corrosión. Se espera que los ladrillos EF3, debido a su baja porosidad total $\left(\mathrm{P}_{\mathrm{T}}=6,18 \%\right)$, su mayor tamaño de grano $\left(\mathrm{S}_{\mathrm{SG}}=245,8 \mu \mathrm{m}\right)$ y una alta relación $\mathrm{C} / \mathrm{S}(\approx 1,50)$ presenten una mayor resistencia al ataque químico de las escorias.

A modo de ejemplo, en la figura 3 se muestra la zona de reacción refractario-escoria perteneciente a las muestras SZ1 y EF2 luego del ensayo de corrosión estática.

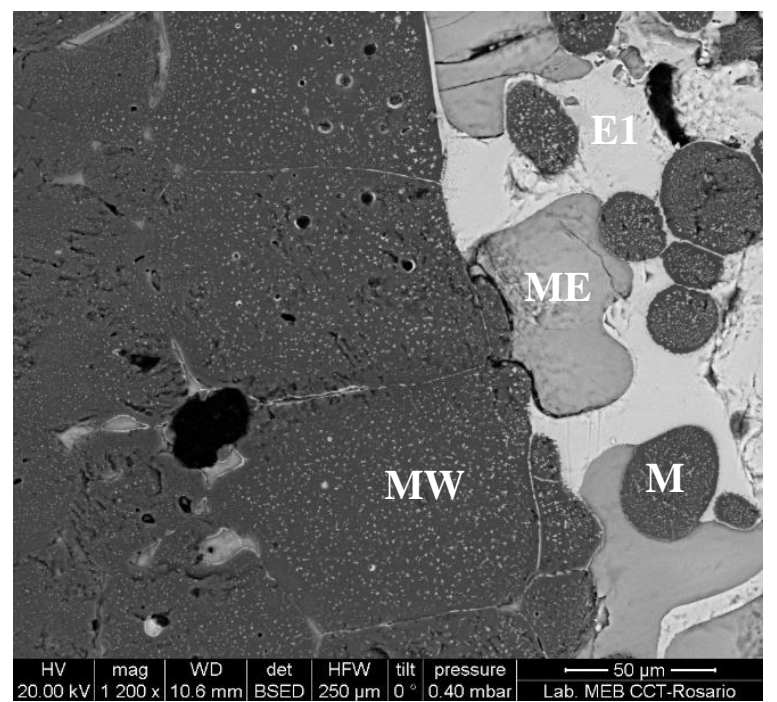

(a)

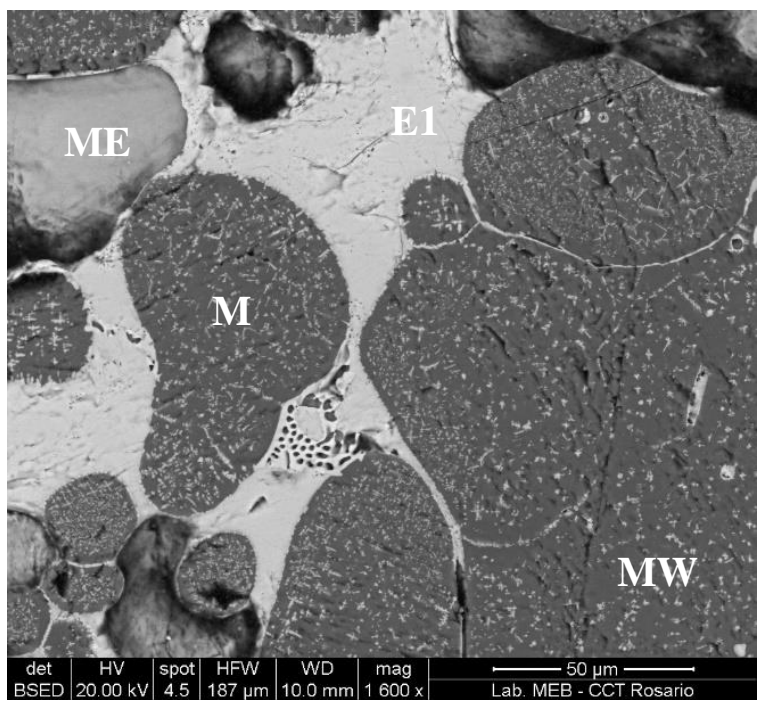

(b)

Figura 3: Imagen de las muestras tras el ensayo de corrosión estática (a) para el ladrillo SZ1 y (b) para el ladrillo EF2.

En la zona de escoria, se puede distinguir claramente granos de $\mathrm{MgO}$ que han sido removidos del ladrillo refractario. El grano marcado como $\mathrm{M}$ es rico en $\mathrm{MgO}$ (71\%), $\mathrm{Fe}_{2} \mathrm{O}_{3}$ (13\%) y $\mathrm{CaO}$ (8\%). Este tipo de granos se consideran originarios del refractario, que han sido atacados por la escoria y "desanclados" del ladrillo, conteniendo en su interior magnesio-wustita ( $\mathrm{MgO}-\mathrm{FeO})$ o magnesio-ferrita $\left(\mathrm{MgO}-\mathrm{Fe}_{2} \mathrm{O}_{3}\right)$. Una capa de $\mathrm{MW}$ se puede distinguir en la región de los granos de $\mathrm{MgO}$ más cercana a la interfaz de reacción escoria-refractario. La zona marcada como E1 corresponde a una fase (líquida a la temperatura del ensayo) que presenta elevado contenido de óxidos de calcio $(\mathrm{CaO} \approx 40 \pm 2 \%)$ y 
hierro $\left(\mathrm{Fe}_{2} \mathrm{O}_{3} \approx 35 \pm 2 \%\right)$. La fase marcada como ME es rica en $\mathrm{CaO}(\approx 56 \pm 2 \%), \mathrm{SiO}_{2}(\approx 33 \pm 2 \%)$ y $\mathrm{MgO}(6 \pm 1 \%)$, de acuerdo a su composición, la fase $\mathrm{ME}$ es asociada a la merwinita ( $\left.3 \mathrm{CaO} . \mathrm{MgO} .2 \mathrm{SiO}_{2}\right)$.

Una cuantificación del grado de ataque se estableció utilizando imágenes de microscopía óptica, donde se determinó, para cada ladrillo, el área penetrada (AP) por la escoria. Para esto se analizó la zona atacada en el fondo del crisol. El área penetrada en las muestras con MgO electrofundida: EF1 $\left(6,97 \mathrm{~mm}^{2}\right)>\mathrm{EF} 2\left(6,63 \mathrm{~mm}^{2}\right)>\operatorname{EF} 3(5,02$ $\left.\mathrm{mm}^{2}\right)$. El área penetrada para las muestras con Mg0 sinterizada resultó ser: SZ2 $\left(13,11 \mathrm{~mm}^{2}\right)>\mathrm{SZ3}\left(8,64 \mathrm{~mm}^{2}\right)>\mathrm{SZ1}$ $\left(8,04 \mathrm{~mm}^{2}\right)$. Para la muestra EFZ con mezclas de magnesias el valor de $\mathrm{AP}=8,45 \mathrm{~mm}^{2}$.

De acuerdo con estos resultados la mayor resistencia al ataque de la escoria es asociada al ladrillo EF3, en acuerdo con lo observado cualitativamente por SEM y con la menor área atacada medida, esto se asocia al alto nivel de pureza, mayor relación C/S y mayor tamaño de grano de la magnesia electrofundida con la que fue fabricado el ladrillo.

En la figura 4 se presentan, a modo de ejemplo, las curvas tensión-deformación de dos ladrillos refractarios obtenidas a temperatura ambiente $\left(\mathrm{T}_{25^{\circ}} \mathrm{C}\right)$ y a $1400^{\circ} \mathrm{C}$. Por cada ladrillo, y en cada condición, se ensayaron dos probetas. A partir de las curvas obtenidas se determinaron los siguientes parámetros mecánicos: (i) resistencia a la fractura $\left(\sigma_{\mathrm{F}}\right)$ tomada como el máximo valor de esfuerzo, (ii) deformación de fractura $\left(\varepsilon_{\mathrm{F}}\right)$, valor de deformación corresponde a la condición de rotura, (iii) módulo de Young $\left(\mathrm{E}_{0,001}\right)$ calculado como la pendiente de la recta secante a la curva trazada desde el origen a un punto de deformación 0,001 pre-establecido, y (iv) energía de fractura $\left(\mathrm{U}_{\mathrm{F}}\right)$ : valor del área debajo de la curva tensión-deformación.

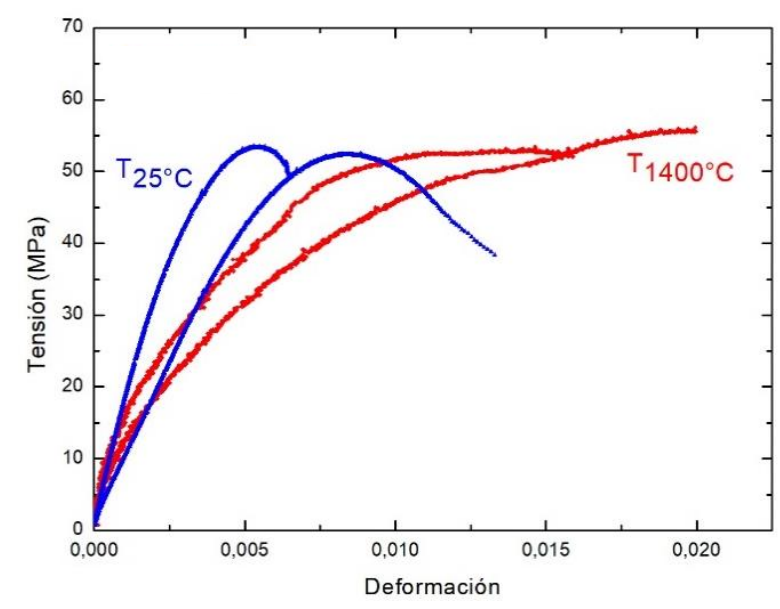

(a)

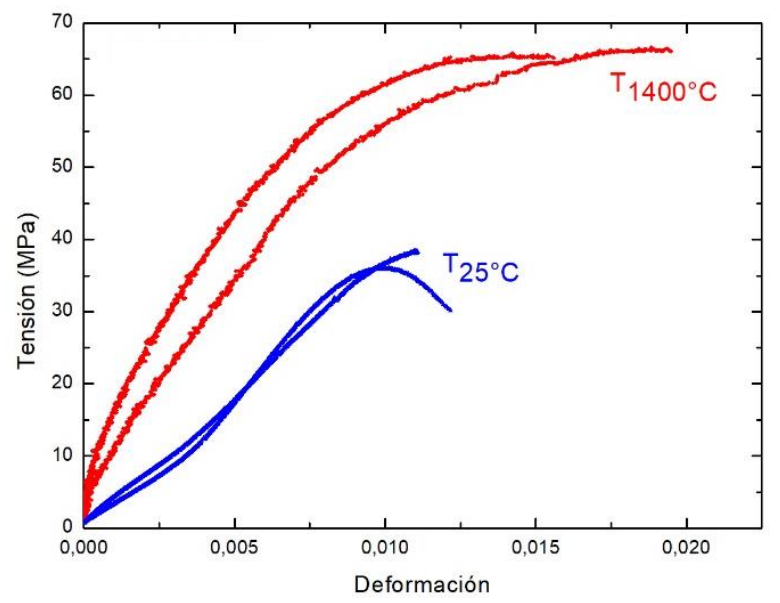

(b)

Figura 4: Curvas tensión-deformación de los materiales refractarios: (a) ladrillo EF2 y (b) ladrillo SZ1.

En estas curvas, es posible visualizar un comportamiento cuasi frágil cuando, para pequeñas deformaciones, las curvas son prácticamente lineales y luego se apartan de la linealidad hasta alcanzar la máxima carga (a partir de la cual se determina la resistencia a la fractura). Desde la máxima tensión $\left(\sigma_{\mathrm{F}}\right)$ hasta la rotura de la muestra, se observa una caída de la tensión al incrementar la deformación, a esto se lo llama "ablandamiento" del material. Podemos observar que las muestras que contiene $100 \%$ de $\mathrm{MgO}$ electrofundida, figura 4 (a), presentan un mayor incremento de elongación $\left(\varepsilon_{\mathrm{F}}\right)$ en caliente, lo cual podría deberse a la formación de fases líquidas en los bordes de grano que aportan plasticidad cuando se encuentran a altas temperaturas. En las muestras con $100 \% \mathrm{MgO}$ sinterizada se observa que los valores de $\sigma_{\mathrm{F}}$ y $\mathrm{U}_{\mathrm{F}}$ a $1400^{\circ} \mathrm{C}$ mejoran notablemente, esto estaría asociado a una mayor densificación de los agregados de $\mathrm{MgO}$ sinterizados respecto de los agregados de $\mathrm{MgO}$ electrofundidos.

El comportamiento mecánico (expresado por los parámetros mecánicos medidos), para todos los ladrillos estudiados, mejora en condiciones de ensayo a $1400^{\circ} \mathrm{C}$. Estos cambios en el comportamiento mecánico se atribuyen a la formación de fases in situ a elevadas temperaturas asociadas a la presencia de antioxidantes (Behera S y Sarkar R, 2016). Estas nuevas fases, aportan cohesión a la estructura, debido a la morfología que adoptan en la matriz y además porque producen en algunos casos densificación (por la propia reacción o porque se forman fases dentro de los poros). Esto último provoca el cierre de fisuras y contribuye al desarrollo de mecanismos de refuerzo (Baudin C. et al, 1999).

El comportamiento térmico evaluado por medio de los ensayos dilatométricos, muestra que se produce una mayor densificación en las muestras que contienen magnesia sinterizada. Debido a que la matriz es la misma en todos los ladrillos, se estima que las mejoras en las propiedades mecánicas $\left(\sigma_{\mathrm{F}}\right.$ y $\left.U_{\mathrm{F}}\right)$ de los ladrillos que se componen con partículas de magnesia sinterizada (SZ1, SZ2, SZ3), estaría asociada a una mayor densificación/sinterización de las mismas respecto de las electrofundidas.

El análisis de los resultados de con DRX sobre cada una de las muestras en estado de recepción, confirma la presencia de silicio ( $\mathrm{Si}$ ) aluminio ( $\mathrm{Al})$, grafito (C) y magnesia (Mg0), en concordancia con el análisis microestructural 
con OM, SEM y EDS, como los componentes principales de todas las muestras contempladas. En base a lo observado en los difractogramas, es posible determinar que la magnitud de los picos de mayor intensidad de la fase grafito en las muestras en estado de recepción tiende a atenuarse en las muestras analizadas luego del ensayo de compresión realizado a $1400^{\circ} \mathrm{C}$. Éste hecho podría atribuirse a la decarburación producida durante el calentamiento. Por otro lado, en base a los resultados obtenidos a partir de los ensayos de difracción de rayos X (DRX) realizados sobre los ladrillos post-ensayo de compresión (a $1400^{\circ} \mathrm{C}$ ), se determina la presencia de magnesia y grafito pero no se logra identificar ni silicio ni aluminio. Sin embargo, es posible confirmar que se ha producido una transformación de fases a partir de la formación de espinela $\left(\mathrm{Al}_{2} \mathrm{MgO}_{4}\right)$ y forsterita $\left(\mathrm{Mg}_{2} \mathrm{SiO}_{4}\right)$ en dichas muestras. La formación de dichas fases es deseable no solo para mejorar la resistencia a la oxidación del carbono, sino también porque pueden aumentar la resistencia mecánica de los materiales refractarios (Xiao J. et al, 2019; Zhu T. et al, 2016).

\section{Conclusiones}

- El ataque de la escoria se produce por la penetración de los granos de $\mathrm{MgO}$ por óxido de $\mathrm{Fe} / \mathrm{Mg}$ para formar solución sólida de magnesio-wustita y magnesio-ferrita, por la infiltración de la escoria a través de los poros, por la reacción de la escoria con las fases silicatos, localizadas en los bordes de grano y puntos triples, generando compuestos de bajo punto de fusión y por la destrucción de las uniones entre granos produciendo un desagregado de los granos de $\mathrm{MgO}$.

- Los parámetros mecánicos $\left(\sigma_{\mathrm{F}}, \mathrm{E}_{0,001} \mathrm{y} \mathrm{U}_{\mathrm{F}}\right)$ medidos a temperatura ambiente son mayores en los ladrillos con $100 \%$ de $\mathrm{MgO}$ electrofundida, el cual presenta las menores porosidades (aparente y real). Mientras que los mayores valores de $\sigma_{\mathrm{F}}$ y $\mathrm{U}_{\mathrm{F}}$ a $1400{ }^{\circ} \mathrm{C}$ se obtienen en los ladrillos conformados con $100 \%$ de $\mathrm{MgO}$ sinterizada, que se asocia a una mayor densificación de los agregados de MgO.

- El incremento de las propiedades mecánicas a $1400{ }^{\circ} \mathrm{C}$ es asociada a la formación de nuevas fases $\left(\mathrm{Al}_{2} \mathrm{MgO}_{4} \mathrm{y}\right.$ $\mathrm{Mg}_{2} \mathrm{SiO}_{4}$ ) debido a la presencia de partículas antioxidantes como aluminio y silicio.

\section{Referencias}

Xiao J., Chen J., Wei Y., Zhang Y., Zhang S., Li N. (2019). Oxidation behaviors of MgO-C refractories with different $\mathrm{Si} / \mathrm{SiC}$ ratio in the $1100-1500{ }^{\circ} \mathrm{C}$ range. Ceramics International, $45,21009-21107$.

https://doi.org/10.1016/j.ceramint.2019.07.086

Behera S., Sarkar R. (2016). Effect of different metal powder anti-oxidants on N220 nano carbon containing low carbon MgO-C refractory: An in-depth investigation. Ceramics International, 42, 18484-18494.

https://doi.org/10.1016/j.ceramint.2016.08.185

Baudín C., Alvarez C., Moore R. E (1999). Influence of Chemical Reactions in Magnesia-Graphite Refractories: I, Effects on Texture and High-Temperature Mechanical Properties. Journal of the American Ceramic Society, 82, 35293538. https://doi.org/10.1111/j.1151-2916.1999.tb02276.x

Zhu T. B., li Y. W., Sang S. B., Jin S. L. (2016). The influence of Al and Si additives on the microstructure and mechanical properties of low-carbon MgO-C refractories. Journal of Ceramic Science and Technology, 07, 127-134. doi: 10.4416/JCST2015-00055 